\title{
Bamboo nodes of the vocal cords
}

\author{
Yue Fan MD, Xingming Chen MD
}

— Cite as: CMAJ 2017 April 18;189:E572. doi: 10.1503/cmaj.160460

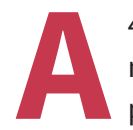

46-year-old man presented with a three-year history of intermittent hoarseness and a six-month history of symmetrical polyarthritis. Fibre optic laryngoscopy showed white transverse submucosal masses resembling bamboo joint nodes on both vocal cords (Figure 1A). The movement of both vocal folds was normal. Biopsy of the lesions showed chronic inflammatory fibrous tissue with immune complex deposition (Appendix 1, available at www. cmaj.ca/lookup/suppl/doi:10.1503/cmaj.160460/-/DC1). Rheumatologic workup revealed an antinuclear antibody titre of 1:80 and a high anti-Jo-1 antibody titre. High-resolution computed tomography of the lungs showed multiple patches of ground-glass opacities consistent with mild interstitial lung disease. The patient did not have muscle weakness or elevated muscle enzyme levels, but his clinical features of inflammatory arthritis, interstitial lung disease and high anti-Jo-1 antibody titre were strongly suggestive of anti-Jo-1 antisynthetase syndrome. The patient received prednisone and cyclophosphamide treatment for eight months. His symptoms of hoarseness and polyarthritis improved substantially over one year of follow-up. Resolution of the vocal cord lesions was confirmed by laryngoscopy (Figure 1B).

Anti-Jo-1 antisynthetase syndrome is a systemic autoimmune disease characterized by myositis, interstitial lung disease, inflammatory polyarthritis and the presence of antisynthetase antibodies, of which anti-Jo-1 is the most common. ${ }^{1}$ Bamboo nodes of the vocal cords were first described in 1993 and have been reported to be associated with a range of autoimmune diseases, including rheumatoid arthritis, systemic lupus erythematosus, autoimmune hepatitis, Hashimoto thyroiditis and systemic sclerosis. ${ }^{2}$ Hoarseness due to the vocal cord lesions may be the presenting symptom in these systemic autoimmune diseases. Biopsy of the lesions using microlaryngoscopy can confirm the diagnosis. Because the lesions have a tendency to recur, treatment of the underlying autoimmune disease is preferred over surgical removal.,3

\section{References}

1. Shinjo SK, Levy-Neto M. Anti-Jo-1 antisynthetase syndrome. Rev Bras Reumatol 2010;50:492-500.

2. Murano E, Hosako-Naito Y, Tayama N, et al. Bamboo node: primary vocal fold lesion as evidence of autoimmune disease. J Voice 2001;15:441-50.

3. Tsunoda K, Soda Y. Hoarseness as the initial manifestation of systemic lupus erythematosus. J Laryngol Otol 1996;110:478-9.

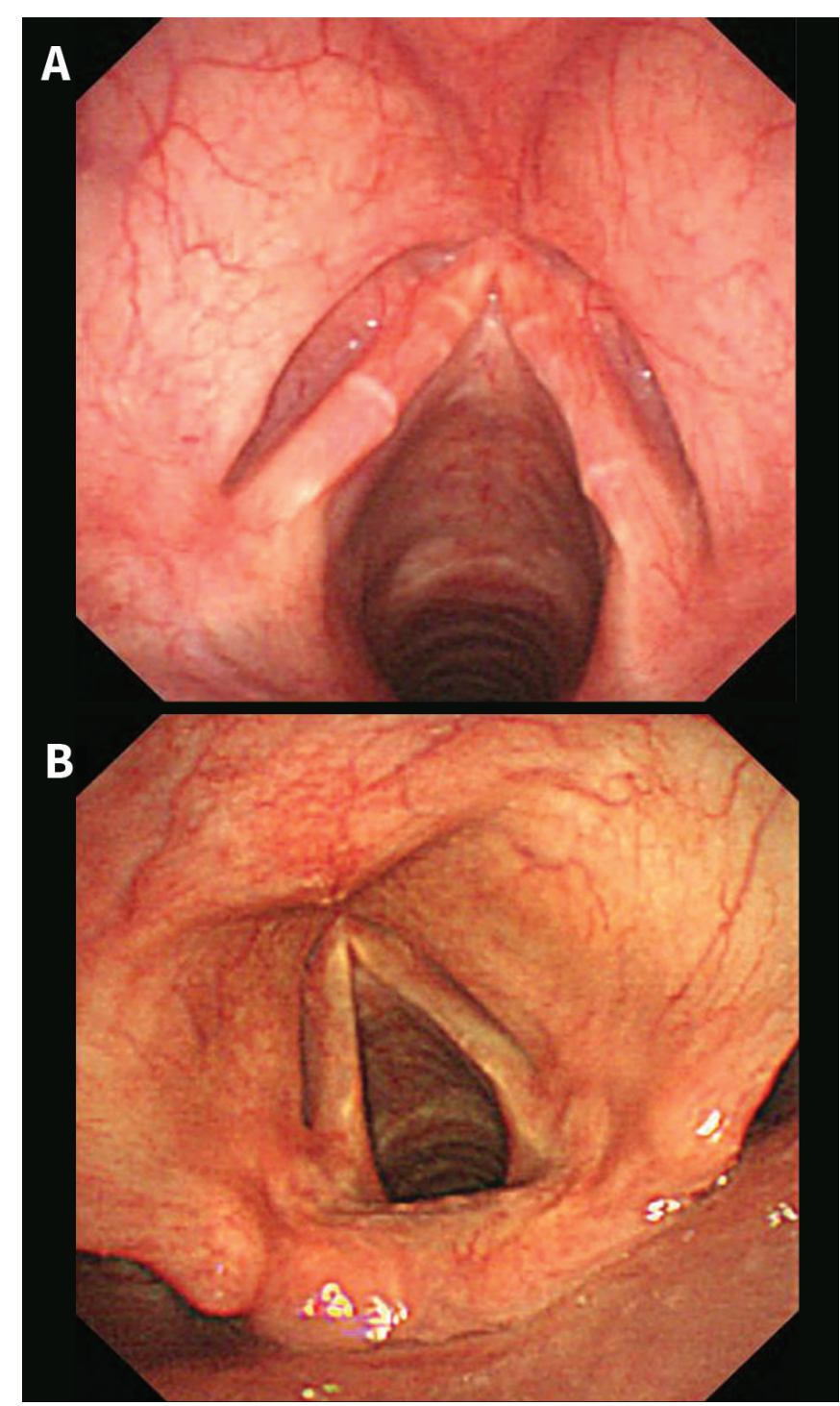

Figure 1: Fibre optic laryngoscopy showing (A) two white transverse submucosal masses resembling bamboo nodes on both vocal cords before treatment in a 46-year-old man with anti-Jo-1 antisynthetase syndrome, and (B) resolution of vocal cord lesions after eight months of treatment.

\section{Competing interests: None declared.}

This article has been peer reviewed.

The authors have obtained patient consent.
Affiliation: Department of Otolaryngology, Peking Union Medical College Hospital, Beijing, China
Correspondence to: Xingming Chen, chenxm@pumch.cn 